\title{
Acute pancreatitis reveals leptospirosis in 18-year- old: a new observation
}

\begin{abstract}
Introduction: Leptospirosis is a globally responsive anthropozoonosis and predominant in tropical countries. It is often under diagnosed because of the frequency of subclinical and anicteric forms. The clinical pictures are extremely polymorphic and can reach all organs. The pancreas is rarely reached and some observations have been reported in the literature.
\end{abstract}

Case report: We describe the case of an 18-year-old patient presenting a leptospirosis revealed by acute pancreatitis and rhabdomyolysis with a good clinical outcome.

Conclusion: leptospirosis has to be included among the etiologies to be evoked in the presence of acute pancreatitis of non-apparent cause.

Keywords: acute pancreatitis, infection, leptospirosis, rhabdomyolysis, zoonosis
Volume 10 Issue 3 - 2019

\section{Krati K, Haraki I, ELyazal S, Jiddi S, Lairani FZ,} Samlani Z, Oubaha S

Department of Hepatology \& gastroenterology, University Hospital Med VI, Morocco

\section{Correspondence: Haraki imane, Department of Hepatology \& gastroenterology, University Hospital Med VI, Morocco, Tel 002126613262 14, Email harakiimane@gmail.com}

Received: June 20, 2018 | Published: May 21, 2019

\section{Introduction}

Leptospirosis is the most widespread zoonosis in the world, caused by a spirochaete called "Leptospira". ${ }^{1}$ The disease is spread worldwide, but its social distribution is unequal. ${ }^{2}$ Rodents and mammals are the main reservoir of this agent, transmitted to humans most often indirectly via contact with soiled water or soil. ${ }^{3,4}$ The clinical picture is polymorphic, ranging from infraclinical forms to severe forms with multivisceral failure and life threatening involvement. It seems that the liver and kidney are the two main organs affected. Other organs can be affected, we mention the eyes, lungs, heart and brain, mainly by a vascular mechanism. ${ }^{5}$ The pancreas is more rarely reached and some sporadic cases have been reported in the literature. We report the case of an 18-year-old patient with a typical picture of acute pancreatitis associated with rhabdomylosis, in whom leptospirosis was diagnosed, with excellent progression under treatment.

\section{Case report}

An 18-year-old young caucasian was admitted to the emergency department in a chart of acute abdominal pain. He was a farmer by profession and lived near livestock in rural areas. He used to swim frequently in dirty water. He had no particular pathological history, including no known pancreatopathy, dysimmunitary pathology or similar cases in the family. The young man had never been bitten by an animal beforehand. The symptomatology started 15 days before admission by the progressive installation of a flu-like syndrome consisting of headache, arthralgia predominant in both knees and myalgia in both lower limbs. The evolution was marked by the aggravation of myalgia which became generalized at the level of the four limbs, becoming disabling and responsible for functional impotence. The patient also had occipital headache but no photophobia, diplopia, visual fog or neck pain. Three days prior to admission, the patient had acute abdominal pain of pancreatic appearance, associated with nausea and bilious vomiting. Everything evolved in a context of deep asthenia and febrile sensations.

The admission examination had found a young conscious patient, anicteric, feverish at $39.1^{\circ} \mathrm{C}$ with tachycardia at $110 \mathrm{beats} / \mathrm{min}$. A sensitivity of the epigastric region with a discrete hepatomegaly at
$14 \mathrm{~cm}$ was found. Functional impotence of the two lower limbs was observed, with a muscular score of $2 / 5$ without abolition of the osteotendinous reflexes and without alteration of the sensitivity. There was no nuchal rigidity or a sign of Kernig or Brudzenski. Examination of the cranial pairs was normal. No other abnormalities were found, especially at the cardio vascular or pleuro pulmonary level. The biological assessment at admission showed predominantly neutrophil leukocytosis at $13850 \mathrm{e} / \mathrm{mm}^{3}$ with thrombocytopenia at $3900 \mathrm{e} / \mathrm{mm}^{3}$. Lipasemia was 9 times normal $(>550 \mathrm{UL} / \mathrm{L})$. The remainder of the report showed: a mild renal failure with a urea at $0.48 \mathrm{~g} / \mathrm{l}$ and a creatinine at $13 \mathrm{mg} / \mathrm{l}$, a rhabdomyolysis syndrome with $\mathrm{CPK}$ elevated to $20000 / \mathrm{L}, \mathrm{LDH}$ at $4272 / \mathrm{L}$ and ASAT at $1044 \mathrm{ui} / 1$. The rest of the balance sheet found ALT at $174 \mathrm{UL} / \mathrm{L}$, acidosis with bicarbonates at $14 \mathrm{mmol} / 1$, corrected serum at $78 \mathrm{mg} / 1$ and normal serum potassium at $3.8 \mathrm{mmol} / 1$ and CRP elevated at $142.5 \mathrm{mg} / \mathrm{ml}$. 1 without significant hyperbilirubinemia or haemostasis disorder. An abdominal ultrasound was performed showing an increased pancreas volume without identifiable obstacle. The abdominal CT confirmed these data by finding a homogeneous increase in pancreatic volume, without identifiable lithiasis, with homogeneous hepatosplenomegaly. Acute pancreatitis stage B of Balthazard was retained. The diet was stopped and the young person was rehydrated with analgesic and gastric protection. The viral serologies B, C, HIV and the phtysiological assessment were negative. The blood cultures were negative. The serology of Martin and Petit was strongly positive. The patient was put on antibiotic with excellent clinical course and complete regression of all symptoms on day 12 of hospitalization. EMG has been delayed due to the progressive improvement of neurological symptoms under specific treatment of leptospirosis. At three months of its release, no abnormal biological balance was found and everything is back in order.

\section{Discussion}

According to the WHO, the incidence of leptospirosis in man is not well known, it would probably be between 0.1 to 1 per 100000 inhabitants per year in temperate climates and 10 or more per 100 000 inhabitants per year in tropical and humid climates. ${ }^{6}$ The main reservoir of leptospires is the renal tubules of animals (rodents, cattle, 
dogs). ${ }^{2}$ It can be described as an emerging occupational zoonosis, as some professions are at higher risk of infection, such as farmers, veterinarians, breeders and abattoir workers. ${ }^{6}$ Our young patient came from a rural area, was a farmer by profession and frequently bathed in freshwater near his home.

Incubation is silent. There are never signs of inflammation at the point of entry. The evolution is typically biphasic with two stages: a first influenza pseudo, and a second multi systemic. Most often, the manifestations are limited to the first phase which is characterized in $75 \%$ of cases by a flu-like syndrome made of fever, myalgia, arthralgia and headache, the case of our patient. The second phase of the disease is usually multisystemic and takes place about ten days after the first phase, most often involving hepato-renal disease. Myalgia is frequent $(60 \%)$ and can be accompanied by rhabdomyolysis of variable severity. ${ }^{2-5}$ Pulmonary, cardiac and neurological manifestations are possible. The hemogram shows neutrophil leukocytosis and thrombocytopenia. Transaminases are most often moderately elevated $(70 \%)$. Impairment of renal function is found in $30 \%$ of cases. Rhabdomyolysis with CPK elevation is typical. ${ }^{2}$ However, the occurrence of massive rhabdomyolysis remains rare.

Taking into consideration the origin and the profession of our patient, the notions of bathing in fresh water, the history of fever, of the flulike syndrome with intense myalgia, the thrombocytopenia, the hyperleucocytosis, the elevation of CPK and aminotransferases, a leptospirosis was strongly suspected. The detection of leptospires on direct examination is theoretically possible under a dark-field microscope in plasma. ${ }^{2}$ In practice this detection is exceptional and the diagnosis does not rely on the identification of bacteria. Serology is the most used exam. The recent development of microagglutination tests (MAT) has improved the sensitivity of diagnostic tests. ${ }^{7}$ It is currently recommended that two microagglutination tests be performed at intervals of 10 days. $^{2}$

In general, the diagnosis of acute pancreatitis is easy in front of an evocative clinical picture and a lipasemia exceeding three times the normal value. ${ }^{8}$ Pancreatic involvement in leptospirosis is rare, probably by vascular mechanism, ${ }^{5}$ and only a few cases have been reported, most often with epigastric pain, nausea or vomiting. ${ }^{7-10}$ In the presence of renal insufficiency and in the absence of a morphological modification of the pancreas on the scanner, the diagnosis of acute pancreatitis in front of a simple increase of the lipasemia becomes more complicated. Authors have reported cases of acute pancreatitis on leptospirosis in the presence of increased lipase and/or amylase levels without morphological changes in the pancreas upon imaging. The clinical picture was often lacking and the majority of these patients had concomitant renal failure, calling into question the virility of the diagnosis. ${ }^{9}$ Our patient had lipase up to 3 times the normal value and the imaging found a pancreas increased in volume homogeneously. The elimination of the most common causes of acute pancreatitis has helped to reinforce the diagnosis of leptospirosis. The management of acute leptospirosis pancreatitis is based on two components: the treatment of the infectious agent and the management of pancreatitis. ${ }^{7}$ Early intravenous antibiotic therapy with penicillin G (5-10 MIU/d) for
10 to 15 days is recommended. ${ }^{2}$ Our patient was put under antibiotics with disappearance of any clinical or biological abnormality. The prophylaxis of the infection imposes a fight against the vectors and a professional prevention. Vaccines and chemoprophylaxis with doxycycline may be proposed for the exposed groups..$^{2-7}$

\section{Conclusion}

Leptospirosis, a global distribution, is common in low socioeconomic areas. In addition to serious or typical forms, the majority of cases are under diagnosed. Some organs are rarely affected, the pancreas for example. The presence of an evocative epidemiological context, a low-level syndrome with headache and feverish myalgia, and biological signs should be a very evocative tripod. The diagnosis should be made quickly so as not to delay the care in order to avoid a potential aggravation.

\section{Acknowledgments}

None.

\section{Conflicts of interest}

Author declares there are no conflicts of interest towards the article.

\section{References}

1. Mazhar M, Kao JJ, Bolger DT Jr. A 23-year-old Man with Leptospirosis and Acute Abdominal Pain. Hawaii JMed Public Health . 2016;75(10):291294.

2. Sersté T, Valla DC. La leptospirose: situation actuelle d'une maladie infectieuse rare. Hépato-Gastro \& Oncologie Digestive. 2006;13(5):353356.

3. Centers for Disease Control and Prevention. Leptospirosis; 2015.

4. Panicker JN, Mammachan R, Jayakumar RV. Primary neuroleptospirosis. Postgrad Med J. 2001;77(911):589-590.

5. Levett PN. Leptospirosis. Clin Microbiol Rev. 2001;14(2):296-326.

6. http://www.who.int/water_sanitation_health/diseases/leptospirosis/fr

7. Chappel RJ, Goris M, Palmer MF, et al. Impact of proficiency testing on results of the microscopic agglutination test for diagnosis of leptospirosis. J Clin Microbiol. 2004;42(12):5484-5488.

8. Smits HL, Eapen CK, Sugathan S. Lateral-flow assay for rapid serodiagnosis of human leptospirosis. Clin Diagn Lab Immunol. 2001;8(1):166-169.

9. Kaya E, Dervisoglu A, Eroglu C, et al. Acute pancreatitis caused by leptospirosis: report of two cases. World $J$ Gastroenterol. 2005;11(28):4447-4449.

10. Silva AP, Burg LB, Locatelli JF, et al. Leptospirosis presenting as ascending progressive leg weakness and complicating with acute pancreatitis. Braz J Infect Dis. 2011;15(5):493-497.

11. Spichler A, Spichler E, Moock M, et al. Acute pancreatitis in fatal anicteric leptospirosis. Am J Trop Med Hyg. 2007;76(5):886-887. 